\title{
A note on AdS cosmology and gauge theory correlator
}

\author{
Souvik Banerjee, ${ }^{a}$ Samrat Bhowmick, ${ }^{b}$ Soumyabrata Chatterjee ${ }^{c}$ \\ and Sudipta Mukherji ${ }^{c}$ \\ ${ }^{a}$ Van Swinderen Institute for Particle Physics and Gravity, University of Groningen, \\ Nijenborgh 4, 9747 AG, The Netherlands \\ ${ }^{b}$ Department of Physics, Indian Institute of Technology Madras, \\ Chennai, 600 036, India \\ ${ }^{c}$ Institute of Physics, Sachivalaya Marg, \\ Bhubaneswar, 751 005, India \\ E-mail: souvik.banerjee@rug.nl, samrat@physics.iitm.ac.in, \\ soumyac@iopb.res.in, mukherji@iopb.res.in
}

ABSTRACT: Using AdS/CFT prescription, we compute two point Yang-Mills correlator on a constant time slice for the Kasner background. Pushing the surface close to the initial singularity, we find, in some cases, the correlator does not develop pole. We further numerically calculate similar correlator where the bulk is a Kasner AdS soliton. We find that the qualitative behaviour of the correlator remains unchanged.

KEYwORDS: Gauge-gravity correspondence, AdS-CFT Correspondence, Spacetime Singularities

ARXIV EPRINT: 1501.06317 


\section{Contents}

1 Introduction 1

2 Scaling solutions $\quad 2$

3 Gauge theory on time-dependent boundary: consequences 3

3.1 Example 1: AdS-Kasner 4

3.2 Example 2: Kasner soliton in AdS $\quad 7$

A Nod from supergravity $\quad 9$

$\begin{array}{ll}\text { B Solving the geodesic equations } & 13\end{array}$

\section{Introduction}

AdS/CFT correspondence relates string theory in AdS space to a supersymmetric $\mathrm{SU}(N)$ Yang-Mills theory living on the boundary of this AdS. The isometry group of the AdS orders the boundary field theory to be conformal. The correspondence can be summarized through the relation:

$$
\frac{L_{\mathrm{AdS}}^{4}}{l_{s}^{4}}=4 \pi g_{\mathrm{YM}}^{2} N \equiv 4 \pi \lambda,
$$

where $l_{s}$ is the string length and $L_{\mathrm{AdS}}$, the curvature length scale of the AdS. $g_{\mathrm{YM}}$ and $N$ denote the coupling constant and the rank of the gauge group of the boundary gauge theory respectively. $\lambda=g_{\mathrm{YM}}^{2} N$ is the t'Hooft coupling. In the limit, $N \rightarrow \infty, g_{\mathrm{YM}} \rightarrow 0$ and $\lambda=$ finite the string theory is classical. When the t'Hooft coupling is large $(\lambda \gg 1)$, the classical string theory reduces to its low energy supergravity limit. Furthermore, a large $\lambda$ makes the field theory to be strongly coupled while the bulk gravity theory remains weakly coupled and hence AdS/ CFT correspondence reduces to a weak-strong duality between a classical gravity theory and a quantum field theory. However, due to strong gravitational fluctuations, this simple limit of the correspondence ceases to exist when the boundary hits, for example, cosmological singularities. Attempts were made to use AdS/CFT to construct time-dependent bulk geometries dual to a boundary field theory well defined even near the cosmological singularity. This is something expected from the fact that AdS / CFT correspondence can be thought of as an example of a more general open stringclosed string duality in string theory. This means, even if the classical supergravity limit of string theory does not exist near singularity, it, in principle, does not prevent one from having a well-defined dual field theory right there. However, the successes in constructing such holographic models have been partial [1] - [5]. 
In this regard, a recent work [7] has provided us with a holographic approach to examining physics near a class of cosmological singularities ${ }^{1}$ The boundary here is given by the Kasner geometry which can appropriately be extended to the bulk. This bulk metric solves five dimensional Einstein equation in the presence of a negative cosmological constant. The work then uses space-like correlators of CFT operators with high enough scaling dimension living on the boundary as a probe. These are "good" probes because the correlators can be well-approximated by exponentiated length of bulk geodesics provided the geodesics go close enough to the cosmological singularity. It is then shown that in certain circumstances the correlator develops a pole in their IR behaviour whenever the geodesic approaches the $t=0$ singularity of the Kasner geometry.

Inspired by this work, in this short note, we revisit the calculation of the correlators. We, however, take a different route of computation. Instead of calculating it on a fixedtime surface, we exploit the underlying scaling symmetry of the Kasner AdS space-time to evaluate the equal-time correlator. The whole process can equivalently be thought of as evaluating the same on an arbitrary space-like surface which finally we push all the way to cosmological singularity and extract the nature of the correlator therein. We find that in the cases where the two points in the boundary are separated in the spatial direction associated with a positive Kasner exponent, ${ }^{2}$ no pole appears in the correlator even near the cosmological singularity. Throughout our analysis, the said asymmetric scaling symmetry of the background metric remains manifest. This in turn enables us to study some general features of the correlator as well.

While the above bulk Kasner-AdS geometry has a Poincare horizon at the core, it is possible to cap it off at finite radial distance by considering a Kasner-AdS soliton instead. We numerically compute the same correlator for this new background and find that the qualitative behaviour remains unchanged.

The plan of the paper is as follows. In the next section, we give a brief account of the cosmological metrics with anisotropic scaling symmetry including the Kasner metric. In section 3, we compute the correlator and study it's properties in a specific cases. In the appendix A, we discuss how the relevant time-dependent geometries can be obtained as near horizon limits of brane-solutions in supergravity. Calculational details are presented in the appendix B.

\section{Scaling solutions}

Several time-dependent solutions of five-dimensional Einstein equations in the presence of negative cosmological constant are known which have anisotropic scaling symmetry of the form

$$
z \rightarrow \lambda z, \quad t \rightarrow \lambda t, \quad x_{i} \rightarrow \lambda^{\left(1-p_{i}\right)} x_{i}(i=1,2 \ldots, n),
$$

where $p_{i}$ 's and $\lambda$ are some constants. Here are some of the examples:

\footnotetext{
${ }^{1}$ Earlier, in [8], boundary correlators were calculated by embedding time dependent backgrounds in AdS.

${ }^{2}$ Given the Kasner conditions, namely, $\sum_{i} p_{i}=1$ and $\sum_{i} p_{i}^{2}=1, p_{i}$ 's being the Kasner exponents, it is guaranteed that we always have at least one positive Kasner exponent.
} 
- The most celebrated example of this comes in the form

$$
d s^{2}=\frac{1}{z^{2}}\left[-d t^{2}+d z^{2}+\sum_{i=1}^{n}\left(a_{i}\right)^{2} d x_{i}^{2}\right],
$$

where $a_{i}=t^{p_{i}} \cdot p_{i}$ are known as Kasner exponents and satisfies the Kasner conditions, namely, $\sum_{i=1}^{n} p_{i}=1$ and $\sum_{i=1}^{n} p_{i}^{2}=1$ in order that (2.2) is a $A d S_{n+2}$ space-time. For $n=3$ and $n=5$ these solutions can also be realized from the perspective of 10 and 11 dimensional supergravity theories as near horizon D3 and $M 5$ brane solutions with Kasner like world volume. Such solutions and their cosmological implications have been studied in detail in [6].

- One of the Kasner conditions can however be relaxed at the expense of introducing matter fields. For instance, there exist AdS solutions with the same metric (2.2) with $\sum p_{i}=1$. For that one needs to introduce a dilatonic scalar field, $\Phi=\lambda \log t$, where $\lambda$ is defined through the modified Kasner condition, $\sum_{i=1}^{n} p_{i}^{2}=1-\frac{\lambda^{2}}{2}$. Such solutions were studied in [9] in the context of probing cosmological singularity through gauge theory duals. These solutions have the scaling symmetry as long as dilaton is shifted by a appropriate constant.

- Finally we shall give example of another class of solutions which has slightly different form than (2.2) but still obeys the scaling relations, (2.1). The metric of the solution takes the form

$$
d s^{2}=\frac{1}{z^{2}}\left[-d t^{2}+\frac{1}{f(z)} d z^{2}+\sum_{i=1}^{n-1} t^{2 p_{i}} d x_{i}^{2}+f(z) d x_{n}^{2}\right]
$$

Here $p_{i}$ 's satisfy Kasner conditions with $p_{n}=0$ and $f(z)=1-\frac{z^{n+1}}{z_{0}^{n+1}}$. The coordinate, $x_{n}$ is an angular coordinate here. These solutions are known as Kasner $A d S_{n+2}$ solitons $[10,11]$ and can be realized in supergravity (for $n=5,7$ ). We put the supergravity origin of these solutions in the appendix A as this is beyond the main point of discussion of this work.

\section{Gauge theory on time-dependent boundary: consequences}

We now turn to the dual gauge theory. As mentioned in the introduction, one of the prime motivations of studying time-dependent AdS geometries have been to understand the physics near cosmological singularity, $t \rightarrow 0$, a sector, otherwise intractable from a direct study.

In order to proceed, one generally computes the space-like two point correlator, $\left\langle\psi\left|\mathcal{O}(x, t) \mathcal{O}\left(x^{\prime}, t\right)\right| \psi\right\rangle$ on a state $|\psi\rangle$ of the strongly coupled Yang-Mills theory residing on the boundary of some of the bulk geometries we discussed in the previous section. When the boundary CFT has a well defined large $N$ limit, the correlator, in the leading order, can be well approximated by

$$
\left\langle\psi\left|\mathcal{O}(x) \mathcal{O}\left(x^{\prime}\right)\right| \psi\right\rangle=e^{-m \mathcal{L}_{\text {reg }}\left(x, x^{\prime}\right)},
$$


provided the operator, $\mathcal{O}$ has a high scale dimension, $\Delta=\frac{d}{2}+\sqrt{\frac{d^{2}}{4}+m^{2}}, d$ being the boundary dimension. Here $\mathcal{L}_{\text {reg }}$ is the regularized length of the geodesic whose end points are fixed at boundary points, $x$ and $x^{\prime}$. With this definition in mind, in the rest of this section we shall compute two examples. First we shall revisit the example of Kasner AdS space-time and then we shall move on to solitonic Kasner-AdS. We will show how some generic features of the result emerge as a consequence of the underlying scaling symmetry of those solutions. For Kasner-Ads soliton, owing to the complication due to the background geometry, we compute the correlator numerically and compare the result with that of Kasner-AdS.

\subsection{Example 1: AdS-Kasner}

We would like to compute $\left\langle\mathcal{O}\left(x_{1}^{\prime}, t_{0}\right) \mathcal{O}\left(x_{1}{ }^{\prime \prime}, t_{0}\right)\right\rangle$ where the bulk geometry is given by $(2.2)$ with $a_{i}=t^{p_{i}}$. This is a correlator along $x_{1}$ direction with two boundary points at $x_{1}^{\prime}, x_{1}{ }^{\prime \prime}$ computed at a fixed time $t=t_{0}$. Corresponding space-like geodesic must then have two fixed end points $x_{1}^{\prime}, x^{\prime \prime}{ }_{1}$ at the boundary $z=0$ at time $t=t_{0}$. For this particular calculation, therefore, the other boundary directions $x_{i}, i \neq 1$ are irrelevant. For the moment, we work with a general scale factor $a_{1}(t)$ along $x_{1}$. Later, we will use the explicit form $a_{1}=t^{2 p_{1}}$ for $p_{1}>0$.

Calling $x_{1}$ as $x$ and $a_{1}$ as $a$ for notational simplicity, the geodesic equations for (2.2) are given by

$$
\begin{aligned}
x^{\prime \prime}+2 \frac{a^{\prime}}{a} x^{\prime}-a a^{\prime} x^{\prime 3} & =0, \\
z z^{\prime \prime}+{z^{\prime}}^{2}+{x^{\prime}}^{2} a^{2}-a a^{\prime} z z^{\prime} x^{\prime 2}-1 & =0 .
\end{aligned}
$$

Here, we have taken time as a parameter and derivatives are with respect to time.

General solutions of these equations can be written as

$$
x(t)= \pm \int \frac{a\left(t^{*}\right) d t}{a(t) \sqrt{a^{2}\left(t^{*}\right)-a^{2}(t)}}
$$

and

$$
z=+\sqrt{-2 \int d t\left[\frac{a(t)}{\sqrt{a^{2}\left(t^{*}\right)-a^{2}(t)}}\left(\int^{t} d t^{\prime} \frac{a\left(t^{\prime}\right)}{\sqrt{a^{2}\left(t^{\prime *}\right)-a^{2}\left(t^{\prime}\right)}}\right)\right]} .
$$

To write (3.3) in this form, we have used the fact that there is a turning point of the geodesic in the bulk and at that point $d x / d t$ diverges. For the solution above, we have taken the point to be $t=t^{*}$.

Given a functional form for $a(t)$, one would then try to integrate the left hand side of the above equations. In this process, three integration constants would appear. However, all of these can be fixed by boundary conditions. The constant appearing from (3.3) can be set to zero by using $x \rightarrow x+$ constant symmetry of the metric. Other two constants arise from the two integrations in (3.4). Both of them can be fixed - (1) by demanding $d z / d x=0$ at the turning point of the geodesic in $z-x$ plane and (2) by requiring $z=0$ for $t=t_{0}$. 
We now argue that if the metric has the scaling symmetry (2.1), then the constant $a\left(t^{*}\right)$ can be scaled away. Taking $a(t)=t^{p}$ and defining new coordinates $\bar{z}, \bar{t}$ and $\bar{x}$ as

$$
z=t^{*} \bar{z}, \quad t=t^{*} \bar{t}, \quad x=t^{* 1-p} \bar{x}
$$

we can re-write (3.3) and (3.4) as

$$
\bar{x}(\bar{t})= \pm \int\left[\frac{d \bar{t}}{\overline{t^{p}} \sqrt{1-\bar{t}^{2 p}}}\right]
$$

and

$$
\bar{z}(\bar{t})=+\sqrt{-2 \int d \bar{t}\left[\frac{\bar{t}^{p}}{\sqrt{1-\bar{t}^{2 p}}}\left(\int^{\bar{t}} d \bar{t}^{\prime} \frac{\bar{t}^{\prime p}}{\sqrt{1-\bar{t}^{\prime 2 p}}}\right)\right]} .
$$

With this, (3.6) and (3.7) can be easily integrated. This gives, for generic $p$

$$
\bar{x}(\bar{t})=\frac{\bar{t}^{1-p}}{1-p}{ }_{2} F_{1}\left(\frac{1}{2}, \frac{1-p}{2 p}, \frac{1+p}{2 p}, \vec{t}^{2 p}\right)-\frac{\sqrt{\pi} \Gamma\left(\frac{1-p}{2 p}\right)}{(1-2 p) \Gamma\left(\frac{1-2 p}{2 p}\right)},
$$

and

$$
\begin{aligned}
\bar{z}(\bar{t})=\left[\vec{t}^{2}\left[1-{ }_{3} F_{2}\left(\left\{1, \frac{1}{2 p}, \frac{1}{p}\right\},\left\{\frac{1}{2}+\frac{1}{2 p}, 1+\frac{1}{p}\right\}, \vec{t}^{2 p}\right)\right]\right. \\
\left.+\frac{4 \sqrt{\pi} p \Gamma\left(\frac{1+p}{2 p}\right) \bar{t}^{1-p}}{(1-2 p) \Gamma\left(\frac{1-2 p}{2 p}\right)}\left[{ }_{2} F_{1}\left(\frac{1}{2}, \frac{1-p}{2 p}, \frac{1+p}{2 p}, \vec{t}^{2 p}\right)-\sqrt{1-\bar{t}^{2 p}}\right]+c\right]^{\frac{1}{2}} .
\end{aligned}
$$

Here, $c$ is a constant which can fixed using $\bar{z}=0$ for $\bar{t}=\bar{t}_{0} .{ }_{2} F_{1}$ and ${ }_{3} F_{2}$ are the hypergeometric function and the generalized hypergeometric function respectively. For some specific values of $p$, the solutions however simplify. In appendix $\mathrm{B}$, we provide a way to solve (3.2) and get to these results.

Having reached this far, we proceed to find the geodesic length. For the correlator, $\left\langle\mathcal{O}\left(x, t_{0}\right) \mathcal{O}\left(-x, t_{0}\right)\right\rangle$, we first need to calculate the integral (3.1)

$$
\mathcal{L}=\int \frac{2 d t}{z}\left[\sqrt{-1+\left(\frac{d z}{d t}\right)^{2}+t^{2 p}\left(\frac{d x}{d t}\right)^{2}}\right],
$$

with appropriate limits. Now, as for the lower limit, the turning point of the geodesic is at $t=t^{*}$. In terms of scaled time, it is at $\bar{t}=1$. For the upper limit, we note that the correlator is being calculated at a constant $t=t_{0}$ slice. This, in terms of scaled variable, is $\bar{t}=\bar{t}_{0}$. We further need to UV-regulate the integral by introducing a cut-off, $\bar{\delta}$. The geodesic length is therefore

$$
\mathcal{L}=\int_{\bar{t}=1}^{\bar{t}=\bar{t}_{0}-\bar{\delta}} \frac{2 d \bar{t}}{\bar{z}}\left[\sqrt{-1+\left(\frac{d \bar{z}}{d \bar{t}}\right)^{2}+\bar{t}^{2 p}\left(\frac{d \bar{x}}{d \bar{t}}\right)^{2}}\right] .
$$

In general, $\mathcal{L}$ is infinite. In order to render it finite, we need to subtract, from $\mathcal{L}$, the equivalent AdS part. This removes the $\bar{\delta} \rightarrow 0$ singularity in the geodesic length. Consequently, the regulated $\mathcal{L}$ will only depend on $\bar{t}_{0}$. 
Above observation, in turn, means that the gauge theory correlator has the form

$$
\left\langle\mathcal{O}\left(x, t_{0}\right) \mathcal{O}\left(-x, t_{0}\right)\right\rangle=e^{-m \mathcal{L}_{\text {reg }}}=f\left(\bar{t}_{0}\right),
$$

for some function $f$. For general $p$, we are unable to evaluate this function analytically. Nevertheless, numerically it can be calculated. We provide our results later. However, for some values of $p$, expressions simplify and analytic computations can be done. As an illustrative example, we do it for $p=1 / 3$. Results are given below.

$$
\begin{aligned}
& \bar{z}(\bar{t})=\sqrt{3\left(\bar{t}^{\frac{4}{3}}-\bar{t}_{0}^{\frac{4}{3}}\right)+\left(\bar{t}^{2}-\bar{t}_{0}^{2}\right)} \\
& \bar{x}(\bar{t})= \pm 3 \sqrt{1-\bar{t}^{\frac{2}{3}}}
\end{aligned}
$$

The geodesic length turn out to be

$$
\begin{aligned}
\mathcal{L} & =\int_{\bar{t}=1}^{\bar{t}_{0}-\bar{\delta}} d \bar{t} \frac{2 \bar{t}^{\frac{1}{3}} \sqrt{1-\bar{t}_{0}^{\frac{2}{3}}}\left(2+\bar{t}_{0}^{\frac{2}{3}}\right)}{\sqrt{1-\bar{t}^{\frac{2}{3}}}\left(3 \bar{t}^{\frac{4}{3}}+\bar{t}^{2}-3 \bar{t}_{0}^{\frac{4}{3}}-\bar{t}_{0}^{2}\right)} \\
& =2 \tanh ^{-1}\left[\frac{\sqrt{1-\left(\bar{t}_{0}-\bar{\delta}\right)^{\frac{2}{3}}}\left(2+\left(\bar{t}_{0}-\bar{\delta}\right)^{\frac{2}{3}}\right)}{\sqrt{1-\bar{t}_{0}^{\frac{2}{3}}}\left(2+\bar{t}_{0}^{\frac{2}{3}}\right)}\right] .
\end{aligned}
$$

Finally, to obtain the regularized length, we need to subtract appropriate AdS contribution. Therefore,

$$
\begin{aligned}
\mathcal{L}_{\text {reg }} & =\mathcal{L}-2 \log \left[\frac{\bar{t}_{0}^{\frac{1}{3}}}{\bar{z}\left(\bar{t}_{0}-\bar{\delta}\right)}\right] \\
& =\log \left[\frac{4\left(4-\bar{t}_{0}^{2}-3 \bar{t}_{0}^{\frac{4}{3}}\right)}{\bar{t}_{0}^{\frac{2}{3}}}\right] .
\end{aligned}
$$

Therefore, we find that $f\left(\bar{t}_{0}\right)$ goes to zero as we take $\bar{t}_{0} \rightarrow 0$.

As we mentioned previously, for arbitrary $p$, it is not possible to evaluate the correlator analytically. However, it is straightforward to carry out a numerical computation. The result is shown in figure 1. Indeed, we find the correlators do not pick up singularities as we take $\bar{t}_{0} \rightarrow 0$.

Having computed the correlator for $p>0$, we proceed to make some general remarks about the correlator. First, let us notice that we can re-write (3.12) as

$$
\left\langle\mathcal{O}\left(t^{*(1-p)} \bar{x}, t^{*} \bar{t}_{0}\right) \mathcal{O}\left(-t^{*(1-p)} \bar{x}, t^{*} \bar{t}_{0}\right)\right\rangle=f\left(\bar{t}_{0}\right) .
$$

However, since $t^{*}$ is a free parameter, we are free to choose it. Let us take $t^{*}=\bar{t}_{0}^{-1}$. Then the correlator takes the form

$$
\left\langle\mathcal{O}\left(\bar{t}_{0}^{(p-1)} \bar{x}, 1\right) \mathcal{O}\left(-\bar{t}_{0}^{(p-1)} \bar{x}, 1\right)\right\rangle=f\left(\bar{t}_{0}\right) .
$$




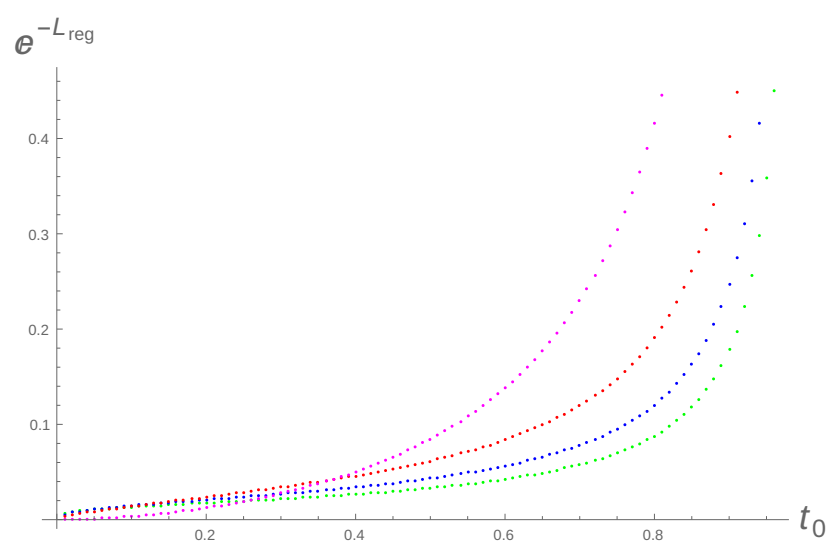

Figure 1. Plot of $e^{-L_{\text {reg }}}$ vs $t_{0}$ for different values of $p$. Magenta, blue and green are for $p=9 / 10,1 / 5$ and $1 / 7$ respectively. For $p=1 / 3$, the numerical and analytical results coincide. The behaviour is shown above in red.

Calling $\mathcal{O}\left(\bar{t}_{0}^{(p-1)} \bar{x}, 1\right)=\tilde{\mathcal{O}}\left(\bar{t}_{0}^{(p-1)} \bar{x}\right)$ and so on we get,

$$
\left\langle\tilde{\mathcal{O}}\left(\bar{t}_{0}^{(p-1)} \bar{x}\right) \tilde{\mathcal{O}}\left(-\bar{t}_{0}^{(p-1)} \bar{x}\right)\right\rangle=f\left(\bar{t}_{0}\right) .
$$

Dependence on the arguments of correlator in this fashion is indeed expected in a scale invariant theory. Note that for $p>0$, as we push the space-like surface close to $\bar{t}_{0}=0$, the separation between the two points in the correlator increases. So we capture the large separation behaviour of the correlator.

The second scaling solution of the previous section has similar metric but there is a non-trivial dilaton. Though this scalar goes to zero at $t=0$, it diverges at a later time - leading to the divergence in Yang-Mills coupling. This however is not a concern for the third scaling solution. This is the Kasner-AdS soliton and we now compute the gauge theory correlator at the boundary of this background.

\subsection{Example 2: Kasner soliton in AdS}

The $A d S_{7}$ Kasner soliton is given by

$$
\begin{aligned}
d s^{2}=\frac{1}{z^{2}}\left[-d \vec{t}^{2}\right. & +\vec{t}^{2 \alpha_{1}} d \bar{x}_{1}^{2}+\vec{t}^{2 \alpha_{2}} d \bar{x}_{2}^{2}+\vec{t}^{2 \alpha_{3}} d \bar{x}_{3}^{2} \\
& \left.+\vec{t}^{2 \alpha_{4}} d \bar{x}_{4}^{2}+\left(1-\frac{z^{6}}{z_{0}^{6}}\right) d \bar{\theta}^{2}+\left(1-\frac{z^{6}}{z_{0}^{6}}\right)^{-1} d z^{2}\right]
\end{aligned}
$$

This is precisely the $n=5$ case of (2.3). The details of derivation of this form is given in (A.20) of the appendix A.

As before, without any loss of generality, we can consider equal time correlators where the boundary points are separated only in $x_{1}$ direction. The geodesic equations are:

$$
\begin{aligned}
-\frac{2 \dot{z}}{z}+\alpha t^{-1+2 \alpha} \dot{x}^{2} & =f(t), \\
\ddot{x}+\frac{2 \alpha}{t} \dot{x}-\frac{2}{z} \dot{x} \dot{z} & =f(t) \dot{x}, \\
\frac{\left(z^{6}-z_{0}^{6}\right)\left(-\dot{x}^{2}\left(z^{6}-z_{0}^{6}\right) t^{2 \alpha}+z^{6}+z z_{0}^{6} \ddot{z}-z_{0}^{6}\right)+\dot{z}^{2}\left(z_{0}^{6}-4 z^{6}\right) z_{0}^{6}}{z_{0}^{6}\left(z^{7}-z z_{0}^{6}\right)} & =f(t) \dot{z},
\end{aligned}
$$


where for notational simplicity we have denoted $x_{1}$ as $x$, avoided the bars from the variables and also called $\alpha_{1}$ as $\alpha$. In the above equations, $f(t)$ is a function of $t$.

Now substituting $f(t)$ from (3.20) into (3.21) and (3.22) we obtain:

$$
\begin{aligned}
& \ddot{x} t=\alpha \dot{x}\left(-2+t^{2 \alpha} \dot{x}^{2}\right), \\
& \begin{aligned}
\left(z^{6}-z_{0}^{6}\right) & \left(z^{6}+z_{0}^{6} z \ddot{z}-z_{0}^{6}\right)-\dot{z}^{2} z_{0}^{6}\left(2 z^{6}+z_{0}^{6}\right) \\
& -\dot{x}^{2}\left(z^{6}-z_{0}^{6}\right) t^{2 \alpha-1}\left(t\left(z^{6}-z_{0}^{6}\right)+\alpha z \dot{z} z_{0}^{6}\right)=0 .
\end{aligned}
\end{aligned}
$$

We further concentrate on the case $\alpha=\frac{1}{3}$ to see a parallel with the case of the Kasner example we considered in the previous subsection. For other positive $\alpha$, qualitative behaviour of the correlator remains same. Equation (3.23) can be solved analytically. We substitute the solution in (3.24) and re-express the $z$-equation as a differential equation in $x$.

$$
\begin{aligned}
\left(x^{2}-9\right)^{3} z^{12}+z_{0}^{12}\left[81\left\{\left(x^{2}-9\right) z z^{\prime \prime}+\left(x^{2}-9\right) z^{\prime 2}-2 x z z^{\prime}\right\}+\left(x^{2}-9\right)^{3}\right] \\
+z_{0}^{6} z^{6}\left[-81\left(x^{2}-9\right) z z^{\prime \prime}+162 z^{\prime}\left\{\left(x^{2}-9\right) z^{\prime}+x z\right\}-2\left(x^{2}-9\right)^{3}\right]=0 .
\end{aligned}
$$

Unlike its counterpart in Kasner-AdS, this equation cannot be solved analytically. However we do find numerical solutions implementing the boundary conditions, namely,

- $\frac{d z}{d x}=0$ at the turning point, $t=t^{*}$ of the geodesic.

- $z=0$ at $t=t_{0}$.

Further, the geodesic length can be written as

$$
L=\frac{2}{9} \int_{0}^{x_{0}-\delta} \frac{d x}{z(x)}\left(x \sqrt{9-x^{2}} \sqrt{\frac{81 z_{0}^{6} z^{\prime}(x)^{2}}{x^{2}\left(9-x^{2}\right)\left(z_{0}^{6}-z(x)^{6}\right)}+\frac{9}{x^{2}}-1}\right) .
$$

Here $x_{0}$ is related to the fixed time-slice $t_{0}$ at the boundary through the solution of (3.23).

$$
x_{0}= \pm 3 \sqrt{1-t_{0}^{\frac{2}{3}}} .
$$

Coordinates in (3.26) and (3.27) are all scaled coordinates as per (3.5) so that the turning point is now at $t=1 . \quad \delta$ is a sharp cut-off in $x$ and signifies the UV cut-off near AdS boundary. The singularity $\delta=0$ can however be taken care of by subtracting from it the corresponding length in AdS with the same UV cut-off, $\delta$, namely

$$
L_{\mathrm{AdS}}=2 \log \left[\frac{t_{0}^{\frac{1}{3}}}{z\left(x_{0}-\delta\right)}\right] .
$$

In figure 1 , we plot $e^{-L_{\text {reg }}}$ as a function of $t_{0}$ where

$$
L_{\mathrm{reg}}=L-L_{\mathrm{AdS}}
$$

The results are plotted in figure 2. The correlator qualitatively shows a behaviour similar to the one we argued for the Kasner geometry. We see here that the function $e^{-L_{\text {reg }}}$ 


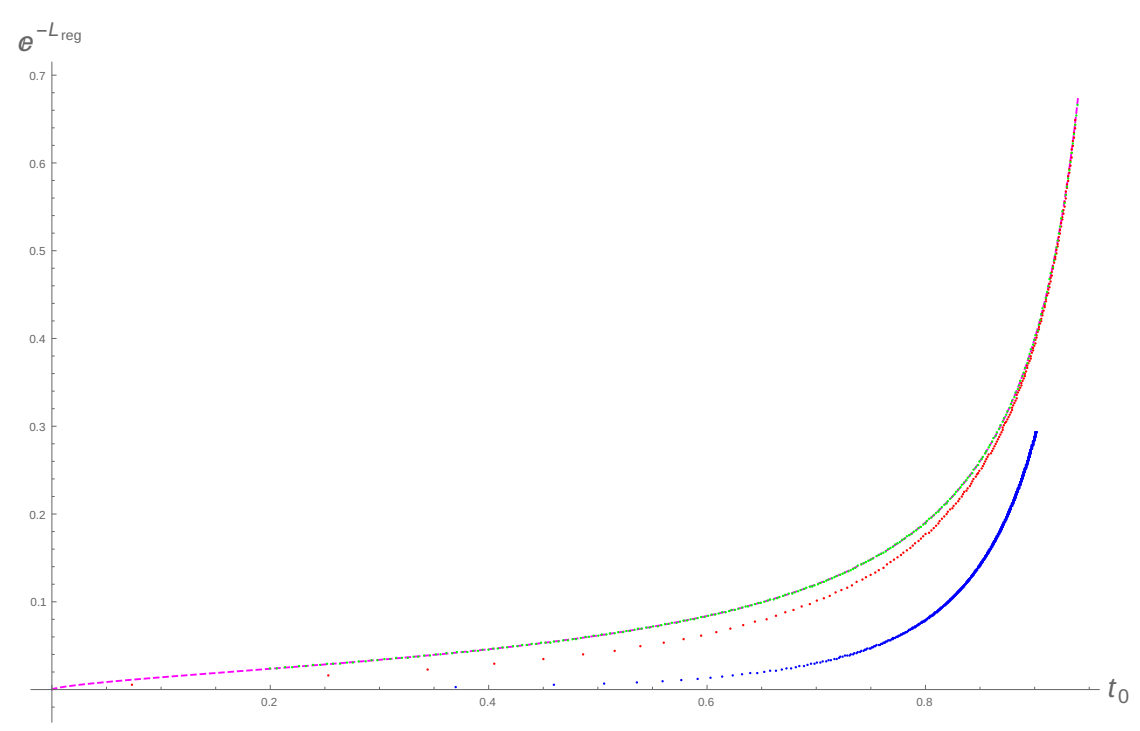

Figure 2. Plot of $e^{-L_{\text {reg }}}$ vs $t_{0}$ for $A d S_{7}$ Kasner soliton. Blue, red and green curves are for $z_{0}=.5,1$ and 10 respectively. For comparison, we also showed, in magenta, $e^{-L_{\mathrm{reg}}}$ for pure $A d S_{7}$ Kasner solution.

goes to zero smoothly as we tune $t_{0} \rightarrow 0$. We further see from the figure that, as $z_{0}$ increases, the plots more and more resemble that of Kasner-AdS. This is expected. As the point $z_{0}$ moves away from the boundary, the correlator sense less of the bulk solitonic geometry.

Finally, we note that in the context of AdS/CFT, it is also possible to construct geometry which breaks the underlying scaling symmetry. For example, one can consider [12]

$$
d s^{2}=\frac{1}{z^{2}}\left[-d t^{2}+d z^{2}+\sum_{i=1}^{n} a_{i}^{2}(t) d x_{i}^{2}+\sum_{j=1}^{m} b_{j}^{2}(t) d \Sigma^{2}\right],
$$

where, $\Sigma$ represents a $m$ dimensional hyperbolic manifold. The metric has cosmological singularities and, owing to the complicated time-dependence of $a$ and $b$, it breaks the scaling symmetry. It would be interesting to compute an appropriate boundary correlator for this geometry. We shall report about such scaling-violating cosmological solutions and their implications in a later issue [13].

\section{Acknowledgments}

We thank Sumit Das, Netta Engelhardt, Suman Ganguly, Thomas Hertog, K. Narayan and Amitabh Virmani for for illuminating discussions and/or fruitful communications.

\section{A Nod from supergravity}

In this appendix we elaborate on how the time-dependent solutions discussed in previous sections inherit a supergravity origin. The solutions we talk about here solves the equations of motion of supergravity theories in 10 and 11 dimensions. 
Solutions in $\boldsymbol{D}=\mathbf{1 0}$. We first review the time-dependent solutions from 10D type IIB supergravity. We primarily concentrate on the Bosonic part of the theory, since for generic time-dependent solutions supersymmetry is explicitly broken. The equations of motion following from the relevant part of standard IIB supergravity action ${ }^{3}$

$$
S_{I I B}=-\frac{1}{16 \pi G_{10}} \int d^{10} x \sqrt{-g}\left(R-\frac{1}{2} \partial^{\mu} \phi \partial_{\mu} \phi-\frac{1}{2 \times 5 !} F_{5}^{2}\right) .
$$

has the forms:

$$
\begin{aligned}
R_{\nu}^{\mu} & =\frac{1}{2} \partial^{\mu} \phi \partial_{\nu} \phi+\frac{1}{2 \times 5 !}\left(5 F^{\mu \xi_{2} \ldots \xi_{5}} F_{\nu \xi_{2} \ldots \xi_{5}}-\frac{1}{2} \delta_{\nu}^{\mu} F_{5}^{2}\right), \\
\partial_{\mu}\left(\sqrt{g} F^{\mu \xi_{2} \ldots \xi_{5}}\right) & =0 \\
\nabla^{2} \phi & =0 .
\end{aligned}
$$

- It was shown in [6] that these equations are solved by the following metric and gauge field configuration:

$$
\begin{aligned}
d s^{2} & =\left(1+\frac{l^{4}}{r^{4}}\right)^{-\frac{1}{2}}\left[-d t^{2}+\sum_{i=1}^{3} t^{2 p_{i}} d x_{i}^{2}\right]+\left(1+\frac{l^{4}}{r^{4}}\right)^{\frac{1}{2}}\left[d r^{2}+r^{2} d \Omega_{5}^{2}\right], \\
F_{t x_{1} x_{2} x_{3} r} & =\frac{2 \sqrt{2} l^{4} t^{p_{1}+p_{2}+p_{3}} r^{3}}{\left(l^{4}+r^{4}\right)^{2}}, \quad F_{i j k l m}=\sqrt{-g} \epsilon_{t x_{1} x_{2} x_{3} r i j k l m} F^{t x_{1} x_{2} x_{3} r} \\
\phi & =0
\end{aligned}
$$

provided the exponents, $p_{i}$, satisfy Kasner conditions, namely

$$
\sum_{i=1}^{3} p_{i}=1 \text { and } \sum_{i=1}^{3} p_{i}^{2}=1
$$

Here, $i, j, k, l, m$ are the indices on $S^{5}$.

In the near horizon limit, $r \rightarrow 0$, the metric reduces to

$$
d s^{2}=-\frac{r^{2}}{l^{2}} d t^{2}+\frac{l^{2}}{r^{2}} d r^{2}+r^{2}\left(t^{2 \alpha} d x^{2}+t^{2 \beta} d y^{2}+t^{2 \gamma} d z^{2}\right)+l^{2} d \Omega_{5}^{2},
$$

with

$$
F_{t x y z r}=\frac{4 t r^{3}}{l^{4}}, \text { giving potential } C_{t x y z}=\frac{t r^{4}}{l^{4}} .
$$

We call it a Kasner- $A d S_{5}$ solution as we discussed under the class of solutions, (2.2). ${ }^{4}$

- Kasner solutions sourced by scalar fields can also be realised, likewise, from the same supergravity set-up. The scalar field profile, however, in this case gets an interpretation of stiff matter on the brane configuration in question.

\footnotetext{
${ }^{3}$ We impose the self-duality condition of the 5-form field strength at the level of equation of motion.

${ }^{4}$ This form is related to the form given in (2.2) by a coordinate transformation, $r=\frac{1}{z}$.
} 
Solutions in $D=11$. In [6] it was also discussed that extremal M5 brane solutions with Kasner-like time dependent scaling of transverse spatial coordinates is a solution of (Bosonic sector of) $D=11$ supergravity with time-dependent gauge fields:

$$
\begin{aligned}
d s^{2}= & \left(1+\frac{l^{3}}{r^{3}}\right)^{-\frac{1}{3}}\left[-d t^{2}+\sum_{i=1}^{5} t^{2 p_{i}} d x_{i}^{2}\right] \\
& +\left(1+\frac{l^{3}}{r^{3}}\right)^{\frac{2}{3}}\left[d r^{2}+r^{2} d \Omega_{4}^{2}\right]
\end{aligned}
$$

along with

$$
F_{t x_{1} x_{2} x_{3} x_{4} x_{5} r}=\frac{3 l^{3} t r^{2}}{\left(l^{3}+r^{3}\right)^{2}}
$$

where the exponents, $p_{i}$, satisfy Kasner conditions, namely

$$
\sum_{i=1}^{5} p_{i}=1 \quad \text { and } \quad \sum_{i=1}^{5} p_{i}^{2}=1 .
$$

In the near horizon limit, i.e. $r \rightarrow 0$, the metric and the non-zero component of the form field reduce to the forms:

$$
\begin{aligned}
d s^{2}= & \frac{r}{l}\left[-d t^{2}+t^{2 \alpha_{1}} d x_{1}^{2}+t^{2 \alpha_{2}} d x_{2}^{2}+t^{2 \alpha_{3}} d x_{3}^{2}+t^{2 \alpha_{4}} d x_{4}^{2}+t^{2 \alpha_{5}} d x_{5}^{2}\right] \\
& +\frac{l^{2}}{r^{2}}\left[d r^{2}+r^{2} d \Omega_{4}^{2}\right], \\
F_{t x_{1} x_{2} x_{3} x_{4} x_{5} r}= & \frac{3 t r^{2}}{l^{3}},
\end{aligned}
$$

Through a change of coordinate,

$$
w^{2}=\frac{r}{l^{3}} .
$$

the metric in (A.10) further takes the form:

$$
d s^{2}=\frac{w^{2}}{4 l^{2}}\left(-d \vec{t}^{2}+\vec{t}^{2 \alpha_{1}} d \bar{x}_{1}^{2}+\vec{t}^{2 \alpha_{2}} d \bar{x}_{2}^{2}+\vec{t}^{2 \alpha_{3}} d \bar{x}_{3}^{2}+\vec{t}^{2 \alpha_{4}} d \bar{x}_{4}^{2}+\vec{t}^{2 \alpha_{5}} d \bar{x}_{5}^{2}\right)+4 l^{2} \frac{d w^{2}}{w^{2}}+l^{2} d \Omega_{4}^{2},
$$

where $\bar{x}_{i}$ and $\bar{t}$ are suitably scaled versions of the coordinates, $x_{i}$ and $t$ respectively. This space we call $K A d S_{7} \times S^{4}$. This solution also belongs to the category discussed under the general form, (2.2).

However, there also exists another negative energy solution of the same supergravity sector. At the level of solutions, such negative energy solutions are obtained through a double analytic continuation of the time and the "p"-th world-volume coordinate of a non-extremal p-brane solution. ${ }^{5}$

$$
t \rightarrow i \theta, \quad x^{p} \rightarrow i t
$$

\footnotetext{
${ }^{5}$ Note, non-extremal M5 branes with Kasner-like world-volume are not solutions of 11D supergravity.
} 
These solutions are known as soliton solutions in literature [10]. In the near horizon limit, one gets the so called AdS solitons which are energetically favoured and hence a more suitable candidate to study the boundary gauge theory.

$t$ being the time coordinate and $\theta$, a periodic angular coordinate, this double analytic continuation amounts to changing the asymptotic topology $R^{p}$ of the parent p-brane configuration to $R^{p-1} \times S^{1}$. Next we look in detail the case in 11-D supergravity when the AdS solitons have time-dependent world-volume.

The generic action for the Bosonic part of $d=11$ supergravity is

$$
S_{11 d}=-\frac{1}{2 \kappa_{11}^{2}} \int d^{11} x \sqrt{-g}\left(R-\frac{1}{48} F_{4}^{2}\right),
$$

The equations of motion arising from (A.14) admits the solitonic solution:

$$
\begin{aligned}
d s^{2}= & \left(1+\frac{l^{3}}{r^{3}}\right)^{-\frac{1}{3}}\left[-d t^{2}+\sum_{i=1}^{4} t^{2 p_{i}} d x_{i}^{2}+\gamma(r) d \theta^{2}\right] \\
& +\left(1+\frac{l^{3}}{r^{3}}\right)^{\frac{2}{3}}\left[\frac{1}{\gamma(r)} d r^{2}+r^{2} d \Omega_{4}^{2}\right]
\end{aligned}
$$

where $\gamma(r)=1-\frac{r_{0}^{3}}{r^{3}}$,

and the gauge field is given by:

$$
F_{\theta x_{1} x_{2} x_{3} x_{4} t r}=\frac{3 \sqrt{l^{3}+r_{0}^{3}} l^{\frac{3}{2}} t r^{2}}{\left(l^{3}+r^{3}\right)^{2}} .
$$

iff the exponents, $p_{i}$ 's satisfy Kasner condition, namely

$$
\sum_{i=1}^{4} p_{i}=1 \quad \text { and } \sum_{i=1}^{4} p_{i}^{2}=1
$$

We call this solution a M5-soliton.

In near horizon limit, the M5-Kasner soliton solution takes the form

$$
\begin{aligned}
d s^{2} & =\frac{r}{l}\left[-d t^{2}+\sum_{i=1}^{4} t^{2 p_{i}} d x_{i}^{2}+\gamma(r) d \theta^{2}\right]+\frac{l^{2}}{r^{2}}\left[\frac{1}{\gamma(r)} d r^{2}+r^{2} d \Omega_{4}^{2}\right], \\
F_{t x_{1} x_{2} x_{3} x_{4} x_{5} r} & =\frac{3 t r^{2}}{l^{3}},
\end{aligned}
$$

We work in the same coordinates defined in (A.11). In these coordinates the metric takes the form

$$
d s^{2}=\frac{w^{2}}{4 l^{2}}\left[-d \bar{t}^{2}+\sum_{i=1}^{4} \vec{t}^{2 p_{i}} d \bar{x}_{i}^{2}+\left(1-\frac{w_{0}^{6}}{w^{6}}\right) d \bar{\theta}^{2}\right]+4 l^{2}\left(1-\frac{w_{0}^{6}}{w^{6}}\right)^{-1} \frac{d w^{2}}{w^{2}}+l^{2} d \Omega_{4}^{2},
$$

where $w_{0}=\frac{r_{0}}{l^{3}} . \bar{x}_{i}$ and $\bar{t}$ are suitably scaled versions of the coordinates, $x_{i}$ and $t$ respectively. Note here, additionally, $\theta$ is also rescaled to $\bar{\theta}$ and hence the period of $\bar{\theta}$ has to be adjusted accordingly. 
Employing the coordinate transformation $\omega=\frac{4 l^{2}}{z}$, the $A d S_{7}$ part of the metric reduces to the familiar form:

$$
\begin{aligned}
d s^{2}=\frac{1}{z^{2}}\left[-d \bar{t}^{2}\right. & +\bar{t}^{2 \alpha_{1}} d \bar{x}_{1}^{2}+\bar{t}^{2 \alpha_{2}} d \bar{x}_{2}^{2}+\bar{t}^{2 \alpha_{3}} d \bar{x}_{3}^{2} \\
& \left.+\bar{t}^{2 \alpha_{4}} d \bar{x}_{4}^{2}+\left(1-\frac{z^{6}}{z_{0}^{6}}\right) d \bar{\theta}^{2}+\left(1-\frac{z^{6}}{z_{0}^{6}}\right)^{-1} d z^{2}\right]
\end{aligned}
$$

This is the same metric as in (2.3).

\section{B Solving the geodesic equations}

Here we discuss a way to solve equations (3.2). It is best to define a new time coordinate $\eta$ such that

$$
\eta=\int \frac{d t}{a^{2}(t)}
$$

The first equation in (3.2) then reduces to

$$
\frac{d^{2} x}{d \eta^{2}}-\frac{1}{a^{3}} \frac{d a}{d \eta}\left(\frac{d x}{d \eta}\right)^{3}=0
$$

Integrating twice, we have

$$
x(\eta)= \pm \int \frac{a(\eta) d \eta}{\sqrt{c_{1} a(\eta)^{2}+1}}+c_{2}
$$

Here $c_{1}$ and $c_{2}$ are the integration constants. Now $c_{1}$ can be fixed using the boundary condition: at the turning point $\eta=\eta^{*}, d x / d t$ or equivalently $d x / d \eta$ is infinity. This gives

$$
c_{1}=-\frac{1}{a^{2}\left(\eta^{*}\right)}
$$

Substituting this in (B.3), we can easily integrate the expression. The result is

$$
x(\eta)=\frac{(1-2 p)^{\frac{1-p}{1-2 p}} \eta^{\frac{1-p}{1-2 p}}}{1-p}{ }_{2} F_{1}\left(\frac{1}{2}, \frac{1}{2}\left(-1+\frac{1}{p}\right), \frac{1+p}{2 p}, \eta^{\frac{2 p}{1-2 p}} \eta^{*-\frac{2 p}{1-2 p}}\right)+c_{2},
$$

where we have used the fact that $a(t)=t^{p}$. Further, $c_{2}$ can be fixed using $x(\eta)=0$ at $\eta=\eta^{*}$. This gives

$$
c_{2}=-\frac{\sqrt{\pi}(1-2 p)^{\frac{p}{1-2 p}} \eta^{* \frac{1-p}{(1-2 p)}} \Gamma\left(-\frac{1}{2}+\frac{1}{2 p}\right)}{\Gamma\left(-1+\frac{1}{2 p}\right)} .
$$

Going over to the $t$ variable, we can write the above equation as

$$
x(t)=\frac{t^{1-p}}{1-p}{ }_{2} F_{1}\left(\frac{1}{2}, \frac{1}{2}\left(-1+\frac{1}{p}\right), \frac{1+p}{2 p},\left(\frac{t}{t^{*}}\right)^{2 p}\right)-\frac{\sqrt{\pi} \Gamma\left(-\frac{1}{2}+\frac{1}{2 p}\right) t^{*(1-p)}}{(1-2 p) \Gamma\left(-1+\frac{1}{2 p}\right)} .
$$

Now we turn to the second equation of (3.2). Defining $K=z z^{\prime}$, we first rewrite it as

$$
\frac{d K}{d t}-a a^{\prime} x^{\prime 2} K+a^{2} x^{2}-1=0
$$


After going to $\eta$ variable, above equation can easily be integrated. This gives

$$
K=\frac{c_{1} a}{\sqrt{c_{1} a^{2}+1}} \int \frac{a^{3}}{\sqrt{c_{1} a^{2}+1}} d \eta+c_{3},
$$

where $c_{3}$ is an integration constant and $c_{1}$ has been defined earlier. More explicitly, we get

$$
K=-\frac{(1-2 p)^{\frac{2 p}{1-2 p}} \eta^{\frac{p}{1-2 p}}}{\sqrt{\eta^{* \frac{2 p}{1-2 p}}-\eta^{\frac{2 p}{1-2 p}}}}\left[\int d \eta \frac{\eta^{\frac{3 p}{1-2 p}}}{\sqrt{\eta^{* \frac{2 p}{1-2 p}}-\eta^{\frac{2 p}{1-2 p}}}}+c_{3}\right] .
$$

It is easy to show that, for $d z / d x$ to vanish at $\eta=\eta^{*}$, the expression inside the brackets has to vanish. This, in turn, fixes $c_{3}$. This gives

$$
\begin{gathered}
K=-\frac{(1-2 p)^{\frac{2 p}{1-2 p}} \eta^{\frac{p}{1-2 p}}}{\sqrt{\eta^{* \frac{2 p}{1-2 p}}-\eta^{\frac{2 p}{1-2 p}}}}\left[( 1 - 2 p ) \eta ^ { \frac { 1 - p } { 1 - 2 p } } \left\{\eta^{* \frac{p}{1-2 p}}{ }^{2} F_{1}\left(\frac{1}{2}, \frac{1}{2}\left(-1+\frac{1}{p}\right), \frac{1+p}{2 p},\left(\frac{\eta}{\eta^{*}}\right)^{\frac{2 p}{1-2 p}}\right)\right.\right. \\
\left.\left.-\sqrt{\eta^{* \frac{2 p}{1-2 p}}-\eta^{\frac{2 p}{1-2 p}}}\right\}-\frac{2 p \sqrt{\pi} \eta^{* \frac{1}{1-2 p}} \Gamma\left(\frac{1+p}{2 p}\right)}{\Gamma\left(\frac{1-2 p}{2 p}\right)}\right] .
\end{gathered}
$$

Now using the property

$$
{ }_{2} F_{1}(a, b, c, z)=(1-z)^{c-a-b}{ }_{2} F_{1}(c-a, c-b, c, z),
$$

the expression for $K$ can be simplified to

$$
\begin{aligned}
K= & (1-2 p)^{\frac{1}{1-2 p}} \eta^{\frac{1}{1-2 p}}\left[1-{ }_{2} F_{1}\left(\frac{1}{2 p}, 1, \frac{1+p}{2 p},\left(\frac{\eta}{\eta^{*}}\right)^{\frac{2 p}{1-2 p}}\right)\right] \\
& +\frac{2 p(1-2 p)^{\frac{2 p}{1-2 p}} \eta^{\frac{p}{1-2 p}} \sqrt{\pi} \eta^{* \frac{1}{1-2 p}} \Gamma\left(\frac{1+p}{2 p}\right)}{\Gamma\left(\frac{1-2 p}{2 p}\right) \sqrt{\eta^{* \frac{2 p}{1-2 p}}-\eta^{\frac{2 p}{1-2 p}}}}
\end{aligned}
$$

This gives

$$
\begin{aligned}
z^{2}= & \int 2(1-2 p)^{\frac{1+2 p}{1-2 p}} \eta^{\frac{1+2 p}{1-2 p}}\left[1-{ }_{2} F_{1}\left(\frac{1}{2 p}, 1, \frac{1+p}{2 p},\left(\frac{\eta}{\eta^{*}}\right)^{\frac{2 p}{1-2 p}}\right)\right] d \eta \\
& +\frac{4 \sqrt{\pi} p(1-2 p)^{\frac{4 p}{1-2 p}} \eta^{* \frac{1}{1-2 p}} \Gamma\left(\frac{1+p}{2 p}\right)}{\Gamma\left(\frac{1-2 p}{2 p}\right)} \int \frac{\eta^{\frac{3 p}{1-2 p}}}{\sqrt{\eta^{* \frac{2 p}{1-2 p}}-\eta^{\frac{2 p}{1-2 p}}}} d \eta+\tilde{c}
\end{aligned}
$$

where $\tilde{c}$ is a constant. Carrying out the integrations, we finally get

$$
\begin{gathered}
z=\left[(1-2 p)^{\frac{2}{1-2 p}} \eta^{\frac{2}{1-2 p}}\left[1-{ }_{3} F_{2}\left(\left\{1, \frac{1}{2 p}, \frac{1}{p}\right\},\left\{\frac{1}{2}+\frac{1}{2 p}, 1+\frac{1}{p}\right\},\left(\frac{\eta}{\eta^{*}}\right)^{\frac{2 p}{1-2 p}}\right)\right]\right. \\
+\frac{4 \sqrt{\pi} p(1-2 p)^{\frac{1+2 p}{1-2 p}} \eta^{*} \frac{1}{1-2 p} \Gamma\left(\frac{1+p}{2 p}\right) \eta^{\frac{1-p}{1-2 p}}}{\Gamma\left(\frac{1-2 p}{2 p}\right)}\left[-\sqrt{\eta^{* \frac{2 p}{1-2 p}}-\eta^{\frac{2 p}{1-2 p}}}\right. \\
\left.\left.+\eta^{* \frac{p}{1-2 p}} 2 F_{1}\left(\frac{1}{2}, \frac{1-p}{2 p}, \frac{1+p}{2 p},\left(\frac{\eta}{\eta^{*}}\right)^{\frac{2 p}{1-2 p}}\right)\right]+\tilde{c}\right]^{\frac{1}{2}} .
\end{gathered}
$$


In terms of variable $t$, we therefore find

$$
\begin{aligned}
z(t)= & {\left[t^{2}\left[1-{ }_{3} F_{2}\left(\left\{1, \frac{1}{2 p}, \frac{1}{p}\right\},\left\{\frac{1}{2}+\frac{1}{2 p}, 1+\frac{1}{p}\right\},\left(\frac{t}{t^{*}}\right)^{2 p}\right)\right]\right.} \\
& \left.+\frac{4 \sqrt{\pi} p \Gamma\left(\frac{1+p}{2 p}\right) t^{* 1+p} t^{1-p}}{(1-2 p) \Gamma\left(\frac{1-2 p}{2 p}\right)}\left[{ }_{2} F_{1}\left(\frac{1}{2}, \frac{1-p}{2 p}, \frac{1+p}{2 p},\left(\frac{t}{t^{*}}\right)^{2 p}\right)-\sqrt{1-\left(\frac{t}{t^{*}}\right)^{2 p}}\right]+\tilde{c}\right]^{\frac{1}{2}} .
\end{aligned}
$$

Equations (B.7) and (B.16) are used in the main text.

Open Access. This article is distributed under the terms of the Creative Commons Attribution License (CC-BY 4.0), which permits any use, distribution and reproduction in any medium, provided the original author(s) and source are credited.

\section{References}

[1] T. Hertog and G.T. Horowitz, Holographic description of AdS cosmologies, JHEP 04 (2005) 005 [hep-th/0503071] [inSPIRE].

[2] B. Craps, T. Hertog and N. Turok, On the quantum resolution of cosmological singularities using AdS/CFT, Phys. Rev. D 86 (2012) 043513 [arXiv:0712.4180] [INSPIRE].

[3] A. Awad, S.R. Das, S. Nampuri, K. Narayan and S.P. Trivedi, Gauge theories with time dependent couplings and their cosmological duals, Phys. Rev. D 79 (2009) 046004 [arXiv: 0807.1517] [INSPIRE].

[4] G. Horowitz, A. Lawrence and E. Silverstein, Insightful D-branes, JHEP 07 (2009) 057 [arXiv: 0904.3922] [INSPIRE].

[5] A. Awad, S.R. Das, A. Ghosh, J.-H. Oh and S.P. Trivedi, Slowly varying dilaton cosmologies and their field theory duals, Phys. Rev. D 80 (2009) 126011 [arXiv:0906.3275] [InSPIRE].

[6] S. Banerjee, S. Bhowmick and S. Mukherji, Anisotropic branes, Phys. Lett. B 726 (2013) 461 [arXiv: 1301.7194] [INSPIRE].

[7] N. Engelhardt, T. Hertog and G.T. Horowitz, Holographic signatures of cosmological singularities, Phys. Rev. Lett. 113 (2014) 121602 [arXiv:1404.2309] [INSPIRE].

[8] K. Koyama and J. Soda, Strongly coupled CFT in FRW universe from AdS/CFT correspondence, JHEP 05 (2001) 027 [hep-th/0101164] [INSPIRE].

[9] A. Awad, S.R. Das, K. Narayan and S.P. Trivedi, Gauge theory duals of cosmological backgrounds and their energy momentum tensors, Phys. Rev. D 77 (2008) 046008 [arXiv: 0711.2994] [INSPIRE].

[10] G.T. Horowitz and R.C. Myers, The AdS/CFT correspondence and a new positive energy conjecture for general relativity, Phys. Rev. D 59 (1998) 026005 [hep-th/9808079] [INSPIRE].

[11] N. Engelhardt and G.T. Horowitz, Entanglement entropy near cosmological singularities, JHEP 06 (2013) 041 [arXiv: 1303.4442] [INSPIRE].

[12] P.K. Townsend and M.N.R. Wohlfarth, Accelerating cosmologies from compactification, Phys. Rev. Lett. 91 (2003) 061302 [hep-th/0303097] [INSPIRE].

[13] S. Banerjee, S. Bhowmick, S. Chatterjee and S. Mukherji, in preparation. 